\title{
RADIATION EFFECT ON MHD STEADY FREE CONVECTION FLOW OF A GAS AT A STRETCHING POROUS SURFACE WITH A UNIFORM FREE STREAM
}

\author{
K Govardhan $^{1 *}$, S Renuka ${ }^{2}$, N Kishan ${ }^{2}$ \\ ${ }^{I}$ Department of Engineering Mathematics, GITAM University, Hyderabad, A.P.India \\ ${ }^{2}$ Department of Mathematics, Osmania University, Hyderabad-500007, A.P, India
}

Received 18 December 2011; received in revised form 17 September 2013

\begin{abstract}
This article investigates the influence of radiation effect on steady free convection flow near isothermal stretching porous sheet in the presence of magnetic field. The governing equations are converted into a system of non-linear ordinary differential equations via a similarity transformation. The resulting system of nonlinear coupled ordinary differential equations are solved numerically by using the Adams-Moulton predictor-corrector method with the shooting method. The numerical results for the velocity and temperature profiles are displayed graphically showing the effect of various values of the flow parameters: radiation parameter $F$, free convection parameter Gr, Magnetic parameter M, Prandtl number Pr, porous parameter $\mathrm{K}$ and the parameter of relative difference between the temperature of the sheet, and the temperature far away from the sheet $\mathbf{r}$. The effect of the radiation and magnetic field parameter on the shear stress, porosity and heat flux are discussed.
\end{abstract}

Keywords: MHD; Radiation; Free Convection; Porous Stretching Sheet.

\section{INTRODUCTION}

The study of the boundary layer behaviour on continuous surface is important because the analysis of such flows finds applications in different areas such as the aerodynamic extrusion of a plastic sheet, the cooling of a metallic plate in a cooling bath, the boundary layer along material handling conveyers, and the boundary layers along a liquid film in condensation processes. As examples on stretched sheets, many metallurgical processes involve the cooling of continues strips or filaments by drawing them through a quiescent fluid and that in the process of drawing, when these strips are stretched. Elbashbeshy [1]

*To whom all correspondence should be addressed. (e-mail: govardhan_kmtm@yahoo.co.in) investigated heat transfer over a stretching surface with variable and uniform surface heat flux subject to injection and suction. Guptha P.S. and Guptha A.S [2] studied the heat and mass transfer corresponding to the similarity solution for the boundary layer over an isothermal stretching sheet subject to blowing or suction. Sakiadis [3], first presented boundary layer flow over a continues solid surface moving with constant speed. Vajravelu and Hadyinicolaou [5] studied the convection heat transfer in an electrically conducting fluid near an isothermal stretching sheet and 
they studied the effect of internal heat generation or absorption.

From the technological point of view, MHD free- convection flows have also great significance for the applications in the fields of stellar and planetary magnetospheres, aeronautics chemical engineering, and electronics. The effects of magnetic field on free convection flow of electrically conducting fluids past a plate studied by many authors such as Soundalgekar [13], Singh et al.[12]. All the above investigations are restricted to MHD flow and heat transfer problems only. However, of late, the radiation effect on MHD flow and heat transfer problems have become more important industrially. At high operating temperature, radiation effect can be quiet significant.

The radiative flows of an electrically conducting fluid with high temperature in the presence of a magnetic field are encountered in electrical power generation, astrophysical flows, solar power technology, space vehicle re-entry, nuclear engineering applications and other industrial areas. The radiation effects on boundary layer flow with and without applying a magnetic field under different situations studied by many investigators for examples: Israel-cookey et al. [9], Mahmoud [10], Hayat et al.[8], Cortel[6] and Sajid and Hayat[11]. The radiation effect on heat transfer of a micropolar fluid through a porous medium Emad M.Abo-Eldahab and Ahmed F.Ghonaim studied[16]. Takhar et al.[4] studied the radiation effects on MHD free convection flow for a non gray-gas past a semi- infinite vertical plate. The radiation effect on steady free convection flow near an isothermal stretching sheet in the presence of a magnetic field is studied by Emad M AboEldahab[17].

In all the above mentioned studiedHer the porosity effects has been neglected. A study of unsteady MHD free convection flow through a porous vertical flat plate immersed in a porous medium in presence of magnetic field with radiation has been analysed by Md.Abdus Samad and Mohd. Mansur Rahman [18]. The effects of thermal radiation and magnetic field on unsteady boundary layer mixed convection flow and heat transfer problem for a vertical porous stretching surface studied by E.M.A Elbashbeshy and D.A.Aldawody[19].The radiation effect on steady free convection flow near isothermal stretching sheet in the presence of a magnetic field is studied by Ahmed Y [14].

The previous work of Ahmed Y [14] neglected the porous mediaum effect. In most of the problems, the combined effect of thermal radiation effectin the presence of porous medium on MHD free convection flow of a Gas at a stretching surface have not been studied. Therefore, the aim of this study is to investigate the effects of radiation on steady free convection flow near isothermal stretching porous sheet in the presence of a magnetic field.

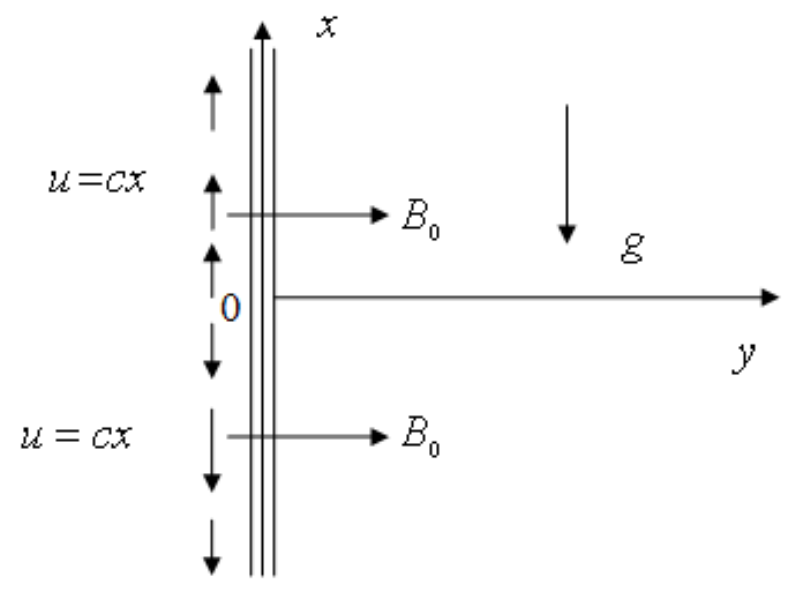

Figure 2. Sketch of the Physical Model

\section{MATHEMATICAL FORMULATION}

Here we consider the flow of an electrically conducting fluid, adjacent to a 
vertical sheet coinciding with the plane $y=0$, where the flow is confined to $y>0$.

Two equal and opposite forces are introduced along the $x$-axis, so that the sheet is stretched keeping the origin fixed. A uniform magnetic field of strength $B_{0}$ is imposed along the $y$-axis. The fluid is considered to be a gray, absorbing emitting radiation but non- scattering medium, gravity acts in the opposite direction to the positive $\mathrm{x}$-axis. The radiative heat flux from the fluid in the $x$-direction is considered negligible in comparison to that in the $y-$ direction. The Rosseland approximation [16] is used to describe the radiative heat flux in the energy equation.

Under the usual boundary layer approximation, the flow and heat transfer in the presence of radiation are governed by the following equations:

$$
\frac{\partial u}{\partial x}+\frac{\partial v}{\partial y}=0
$$

$u \frac{\partial u}{\partial x}+v \frac{\partial u}{\partial y}=v \frac{\partial^{2} u}{\partial y^{2}}+g \beta\left(T-T_{\infty}\right)-\frac{\sigma B_{0}^{2}}{\rho} u-\frac{v}{k} u$

$$
u \frac{\partial T}{\partial x}+v \frac{\partial T}{\partial y}=\frac{k}{\rho c_{p}} \frac{\partial^{2} T}{\partial y^{2}}-\frac{1}{\rho c_{p}} \frac{\partial q_{r}}{\partial y}
$$

where $u$ and $v$ are the velocity components in the $x$ and $y$-directions, respectively, $\mathrm{T}$ is the temperature, $\mathrm{g}$ is the acceleration due to gravity, $v$ is the fluid kinematics viscosity, $\rho$ is the density, $\sigma$ is the electric conductivity, $C_{p}$ is the specific heat at constant pressure, and $q_{r}$ is the radiative heat flux. problem

The boundary conditions of the

$$
\begin{gathered}
u=c x, v=0, T=T_{w} \quad y=0 \\
u \rightarrow u_{\infty}, T \rightarrow T_{\infty} \text { at } y \rightarrow \infty
\end{gathered}
$$

Where $c>0, T_{w}$ is the constant temperature of sheet, $T_{\infty}$ is the temperature far away from the sheet, and $u_{\infty}$ is the free stream velocity. By using the Rosseland approximation [16], we have

$q_{r}=-\frac{4 \sigma^{*}}{3 k^{*}} \frac{\partial T^{4}}{\partial y}$

Where $\sigma^{*}$ is the Stefan- Boltzmann constant and $k^{*}$ is the mean absorption coefficient. By using (5), the energy equation (3) becomes

$u \frac{\partial T}{\partial x}+v \frac{\partial T}{\partial y}=\frac{k}{\rho c_{p}} \frac{\partial^{2} T}{\partial y^{2}}+\frac{4 \sigma^{*}}{3 k^{*} \rho c_{p}} \frac{\partial^{2} T^{4}}{\partial y^{2}}$

where $M=\frac{\sigma B^{2}{ }_{0}}{\rho c}$ is the magnetic parameter $R=\frac{u_{\infty}}{\sqrt{c v}}$ is the Reynolds number,

$G r=\frac{g \beta\left(T_{w}-T_{\infty}\right)}{c u_{\infty}}$ is the free convection parameter,

$\operatorname{Pr}=\frac{\mu c_{p}}{k}$ is the prandtl number,

$\mathrm{F}=\frac{k k^{*}}{4 \sigma^{*} T_{\infty}^{3}}$ is the radiation parameter, $\mu=\rho v$ is the viscosity of the fluid, and $\mathrm{r}=\frac{\left(T_{w}-T_{\infty}\right)}{T_{\infty}}$ is the relative difference between the temperature of the sheet and the temperature far away from the sheet.

Introducing the following non dimensional parameters:

$$
\begin{array}{ll}
\bar{x}=\frac{c x}{u_{\infty}}, & \bar{y}=\frac{c y R}{u_{\infty}}, \quad \bar{u}=\frac{u}{u_{\infty}}, \\
\bar{v}=\frac{v R}{u_{\infty}}, & \theta=\frac{T-T_{\infty}}{T_{w}-T_{\infty}}
\end{array}
$$

We can obtain the governing equation in dimensionless form as (with dropping the bars) 
$\frac{\partial u}{\partial x}+\frac{\partial v}{\partial y}=0$

$u \frac{\partial u}{\partial x}+v \frac{\partial u}{\partial y}=\frac{\partial^{2} u}{\partial y^{2}}+G r \theta-(M+K) u$

$u \frac{\partial \theta}{\partial x}+v \frac{\partial \theta}{\partial y}=\frac{1}{\operatorname{Pr}} \frac{\partial^{2} \theta}{\partial y^{2}}+\frac{4}{3 F \operatorname{Pr}}\left((1+r \theta)^{3} \frac{\partial^{2} \theta}{\partial y^{2}}+3 r(1+r \theta)^{2}\left(\frac{\partial \theta}{\partial y}\right)^{2}\right)$

With the boundary conditions

$$
\begin{aligned}
& u=x, v=0, \theta=1 \quad \text { at } \mathrm{y}=0, \\
& u=1, \quad \theta=0 \text { as } y \rightarrow \infty,
\end{aligned}
$$

Introducing the stream function $\psi$ defined in the usual way

$$
u=\frac{\partial \psi}{\partial y}, \quad v=-\frac{\partial \psi}{\partial x}
$$

Equation (10) can then be written as

$$
\begin{gathered}
\frac{\partial \psi}{\partial y} \frac{\partial^{2} \psi}{\partial x \partial y}-\frac{\partial \psi}{\partial x} \frac{\partial^{2} \psi}{\partial y^{2}}=\frac{\partial^{3} \psi}{\partial y^{3}}-M \frac{\partial \psi}{\partial y}-K \frac{\partial \psi}{\partial y}+G r \theta \\
\frac{\partial \psi}{\partial y} \frac{\partial \theta}{\partial x}-\frac{\partial \psi}{\partial x} \frac{\partial \theta}{\partial y}=\frac{1}{\operatorname{Pr}} \frac{\partial^{2} \theta}{\partial y^{2}}+\frac{4}{3 F \operatorname{Pr}}\left((1+r \theta)^{3} \frac{\partial^{2} \theta}{\partial y^{2}}+3 r(1+r \theta)^{2}\left(\frac{\partial \theta}{\partial y}\right)^{2}\right)
\end{gathered}
$$

And the boundary conditions (10) become

$$
\begin{array}{ll}
\frac{\partial \psi}{\partial y}=x & \frac{\partial \psi}{\partial x}=0, \quad \theta=1, \text { at } y=0 \\
\frac{\partial \psi}{\partial y}=1, & \theta=0 \quad \text { as } y \rightarrow \infty . \quad(13)
\end{array}
$$

Introducing,

$\psi(x, y)=f(y)+x g(y)$,

Substituting the equation (14) in equations (12) and equating the coefficients of $x^{0}$ and $x^{1}$, we obtain the coupled nonlinear ordinary differential equations

$f^{\prime \prime \prime}=f^{\prime} g^{\prime}-g f^{\prime \prime}+(M+K) f^{\prime}-G r \theta$

$g^{\prime \prime \prime}=g^{\prime 2}-g g^{\prime \prime}+(M+K) g^{\prime}$

$\left(3 F+4(1+r \theta)^{3}\right) \theta^{\prime \prime}+3 \operatorname{Pr} F g \theta^{\prime}+12(1+r \theta)^{2} \theta^{\prime 2}=0$

The primes above indicate differentiation with respect to $y$ only. The boundary conditions (13) in view of (14) is reduced to

$$
\begin{aligned}
& f(0)=f^{\prime}(0)=g(0)=g^{\prime}(\infty)=\theta(\infty)=0 \\
& g^{\prime}(0)=\theta(0)=f^{\prime}(\infty)=1
\end{aligned}
$$

The physical quantities interested in this problem are the skin friction coefficient and the Nusselt number, which are defined by

$\tau_{w}=\left.\mu \frac{\partial u}{\partial y}\right|_{y=0} N_{u}=\frac{q_{w}}{k\left(T_{w}-T_{\infty}\right)}$

where $q_{w}=-\left.k \frac{\partial T}{\partial y}\right|_{y=0}$

Using (14), the quantities in (19) can be expressed as

$\tau_{w}=\mu c R\left(f^{\prime \prime}(0)+x g^{\prime \prime}(0)\right), N_{u}=\frac{c R}{u_{\infty}} \theta^{\prime}(0)(20)$

The effect of parameters F, Gr, M, and $\mathrm{Pr}$, on the functions $f^{\prime \prime}, g^{\prime \prime}$ and $\theta^{\prime}$ at the plate surface is tabulated in Table1 for $r=0.05$.

\section{METHOD OF SOLUTION}

Closed form solution for the equation

$$
g(y)=\frac{1}{\sqrt{M+1}}\left[1-e^{-\sqrt{M+1} y}\right]
$$

For the purpose of numerical computation, the differential equations (15) \& (17) are written in the form of a system of first order differential equations. The following transformation variables were used.

$Y_{1}=f \quad ; \quad Y_{2}=f^{\prime} ; \quad Y_{3}=f^{\prime \prime} ; \quad Y_{4}=\theta ; \quad Y_{5}=\theta^{\prime}$

With this substitution, the couple system of equations (15) \& (17) can be written as a first order system as:

$$
\begin{aligned}
& Y_{1}^{\prime}=Y_{2} \\
& Y_{2}^{\prime}=Y_{3} \\
& Y_{3}^{\prime}=Y_{2} g^{\prime}-Y_{3} g+(M+K) Y_{2}-G r Y_{4} \\
& Y_{4}^{\prime}=Y_{5}
\end{aligned}
$$


and

$$
Y_{5}^{\prime}=\frac{-3 \operatorname{Pr} F g Y_{5}+12 r(1+r \theta)^{2} Y_{5}^{2}}{3 F+4(1+r \theta)^{3}}
$$

The boundary conditions (18) give

$Y_{1}(0)=Y_{2}(0)=0, \quad Y_{3}(0)=\alpha, Y_{4}(0)=1$, $Y_{5}(0)=\beta$

The fundamental problem is to find the solution to differential equations (15)-(17) subject to the boundary conditions (18) for the various values of parameter $\alpha, \beta$. In this case choice of missing initial solution is difficult. Assuming initial values for $\alpha$ and $\beta$ the system is solved. An iterative shooting method which uses globally convergent Newton Raphson method is employed. The guess solution is changed in systematic way until correct starting values are determined. The fourth order Adams predictor-corrector method is used to solve the system of equations.

\section{RESULTS AND DISSCUSSION}

The numerical results for the velocity and temperature distribution are shown in Figure 2-10 for different flow field parameters of Prandtl number Pr, free convection parameter $G r$, radiation parameter $F$, the parameter of relative difference between the temperature of the sheet, and the temperature far away from the sheet $r$ and the porous parameter $K$. Figure 2 (a) displays the effect of $\mathrm{Pr}$ and $\mathrm{F}$ on the velocity distribution. It is seen from this Figure that the velocity profile $f^{\prime}$ decreases with the increase of the $F$ and Pr. The effect of $r$ increases the velocity profile $f^{\prime}$, while the velocity profile $f^{\prime}$ decreases with the increase of magnetic field parameter $M$ is observed from Figure 2(b).

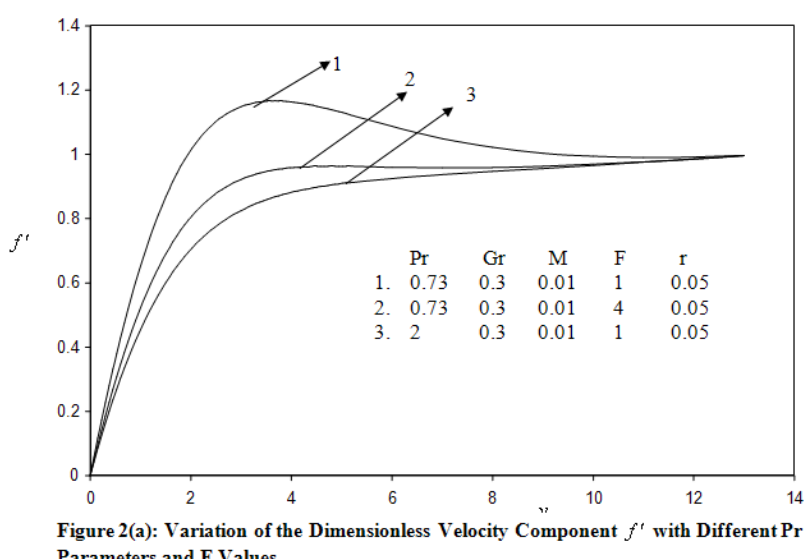

Parameters and F Values

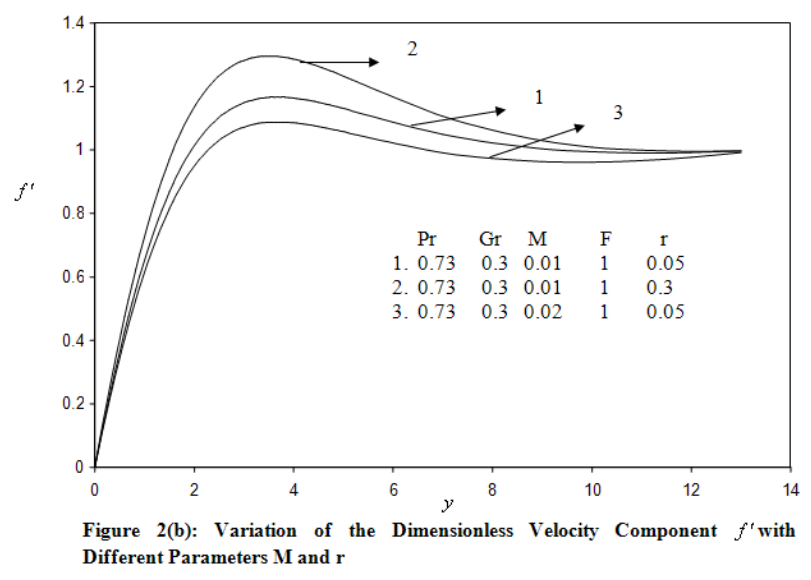

From Figure 3 it is observed that the temperature distribution decreases as $F$ increases as well as Pr increases. It is also noticed that the temperature distribution increases as $r$ increases. This is an agreement with the physical fact that the thermal boundary thickness decreases with the increasing Pr.

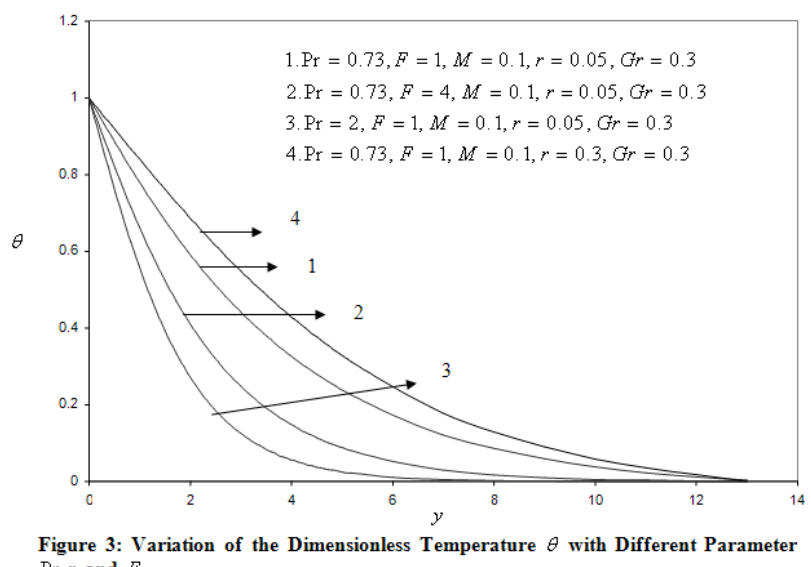
$\mathrm{Pr}, r$, and $F$ 


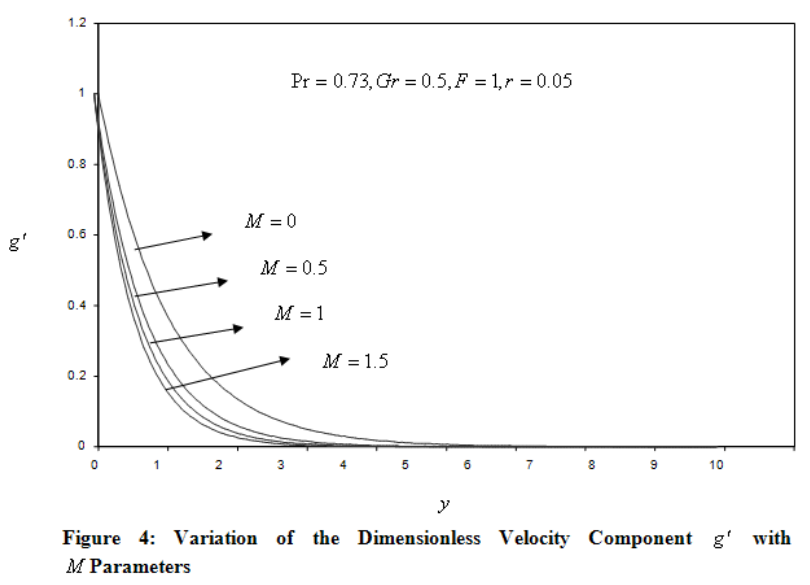

Figure 4 displays the behavior of $g^{\prime}$ with changes in the values of the magnetic field parameter $M$. It is seen, that the $g^{\prime}$ decreases with the increasing the magnetic field parameter $M$. The effects of porous parameter on $f^{\prime}, g^{\prime}$ and $\theta$ are illustrated in Figure 5,6,7,8,9, and 10 .

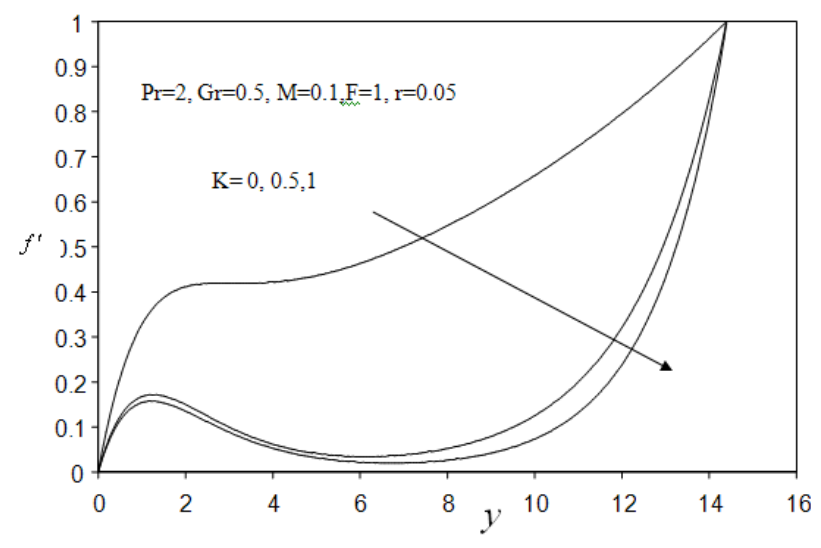

Figure 5: Variation of the Dimensionless Velocity Component $f^{\prime}$ with $\mathbf{K}$ Parameter

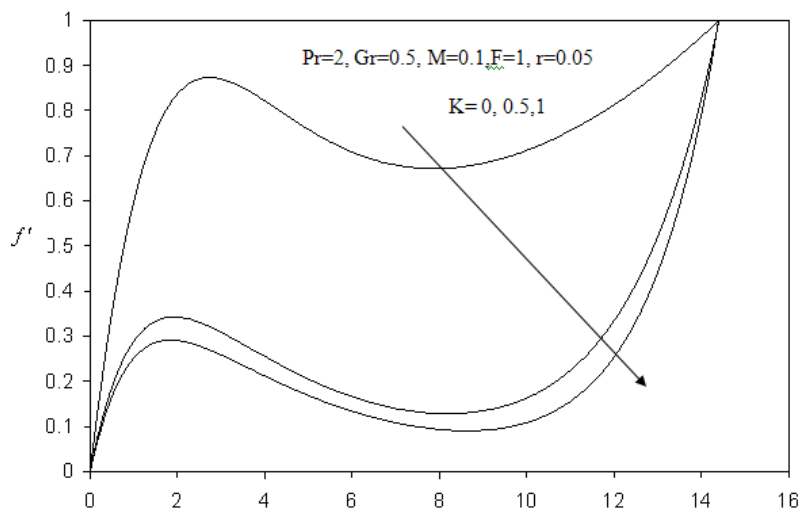

Figure 6: Variation of the Dimensionless Veloc $y$,mponent $f^{\prime}$ with K Parameter
The effect of porous medium on the dimensionless velocity component $f^{\prime}$ is displayed in the Figure 5 and 6 for different Pr values. It is clear from this Figure that the velocity of the fluid is decreases with the increase of the porous parameter. The increasing in fluid temperature due to viscosity is observed to be more pronounced for higher values of $\mathrm{K}$.

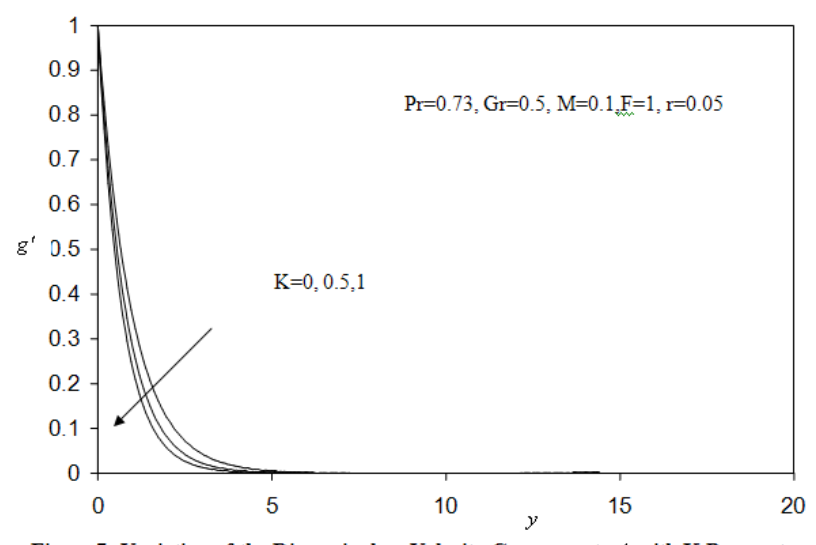

Figure 7: Variation of the Dimensionless Velocity Component $g^{\prime}$ with K Parameter

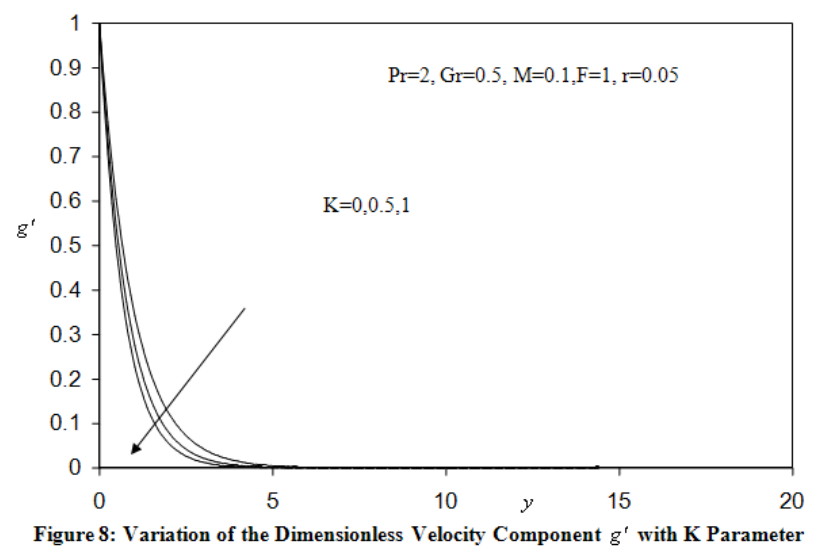

Figure 7 and 8 shown that the effect of porous parameter $\mathrm{K}$ on the velocity profile for different Pr values. It is seen from this figure that the velocity decreases profile increases with the increase of porous parameter K. Figure 9 and 10 shown that the effect of porous parameter on temperature profile for different Pr values. It is seen from this figure that the temperature profile increases with increase of porous parameter $\mathrm{K}$. It can be seen that the velocity profiles as 
well as temperature profiles are higher when $\operatorname{Pr}=0.73$ compared with $\operatorname{Pr}=2$.

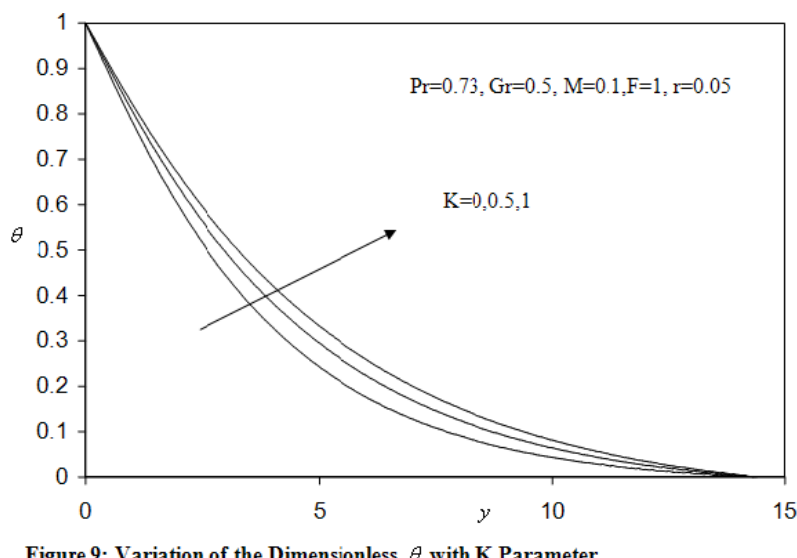

Figure 9: Variation of the Dimensionless $\theta$ with K Parameter

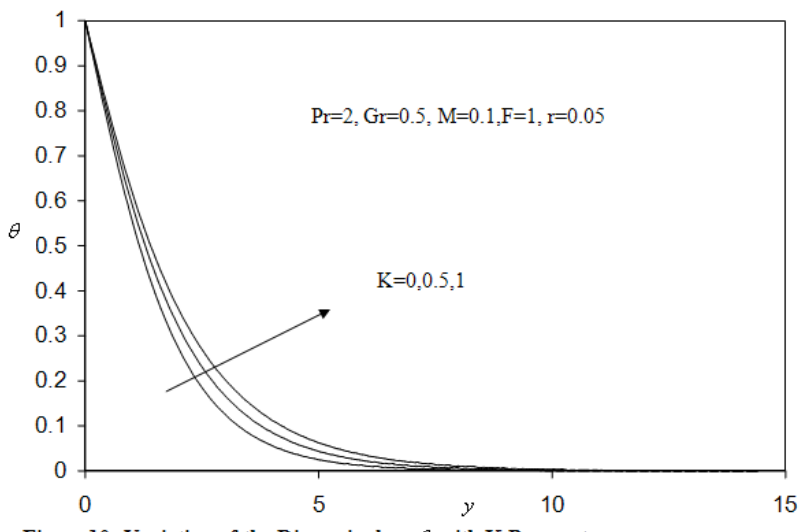

Figure 10: Variation of the Dimensionless $\theta$ with K Parameter

The effect of variation parameter on the skin friction coefficient $f^{\prime \prime}(0)$ is shown in Figure 11 and the heat flux $\theta^{\prime}(0)$ is shown in Figure 12. Figure 11 display the effect of magnetic field parameter $M$ on the skin friction coefficient $f^{\prime \prime}(0)$ with the effect of magnetic parameter $\mathrm{M}$ the $f^{\prime \prime}(0)$ value is decreases. It is noticed that the effect of increasing $M$ is the decrease in the wall temperature gradient $\theta^{\prime}(0)$ is shown in Figure 12. Variation of $f^{\prime \prime}, g^{\prime \prime}, \theta^{\prime}$ at the plate surface with different values of $F, G r, M$ Pr are shown in Table (1)-(2) From these tables, it should be mentioned that the results obtained herein are good agreement with the previous work of Ahmed
$\mathrm{Y}[15]$ when $\mathrm{K}=0$, which gives validation of the present solution.

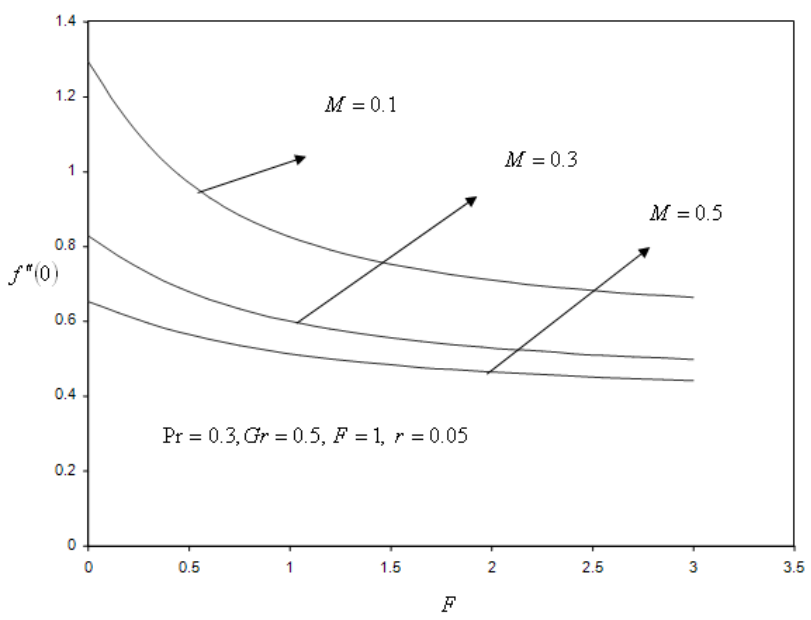

Figure 11: Variation of $f^{\prime \prime}(0)$ with $F$ and $M$ Parameters

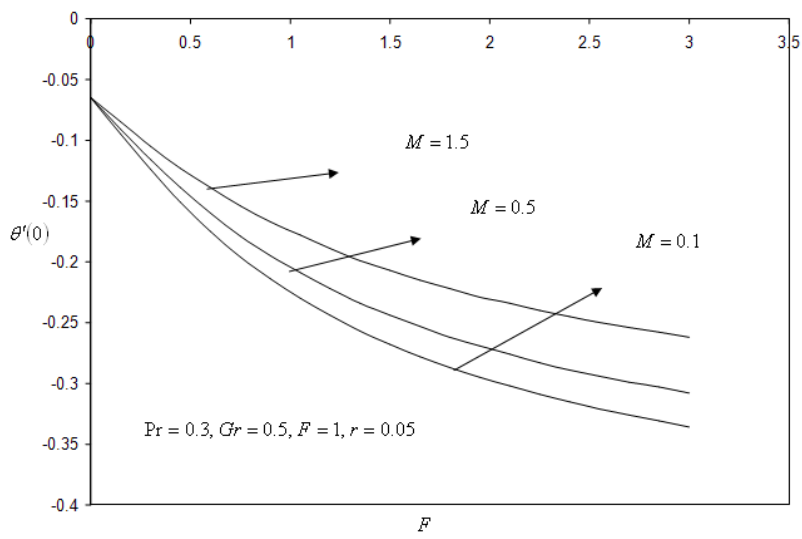

Figure 12: Variation of the Heat Flux $\theta^{\prime}(0)$ with $F$ and $M$ parameters

Table 1: Variation of $f^{\prime \prime}, g^{\prime \prime}, \theta^{\prime}$ at the Plate Surface with $F, G r, M$ and Pr Parameters

\begin{tabular}{cccccccccc}
\hline$F$ & $G r$ & $M$ & Pr & $\begin{array}{l}\text { Ahmed } \\
\mathrm{Y}[15] g^{\prime \prime}(0)\end{array}$ & $\begin{array}{l}g^{\prime \prime}(0) \\
\text { Present }\end{array}$ & $\begin{array}{l}\text { Ahmed } \\
\mathrm{Y}[15] \\
\theta^{\prime}(0)\end{array}$ & $\begin{array}{l}\theta^{\prime}(0) \\
\text { Present }\end{array}$ & $\begin{array}{l}\text { Ahmed } \\
\mathrm{Y}[15] \\
f^{\prime \prime}(0)\end{array}$ & $\begin{array}{l}f^{\prime \prime}(0) \\
\text { Present }\end{array}$ \\
\hline 1 & 0.5 & 0.1 & 0.73 & -1.04771 & -1.049 & -0.224411 & -0.225 & 0.820805 & 0.825 \\
2 & 0.5 & 0.1 & 0.73 & -1.04771 & -1.049 & -0.297402 & -0.298 & 0.703769 & 0.705 \\
3 & 0.5 & 0.1 & 0.73 & -1.04771 & -1.049 & -0.335702 & -0.336 & 0.656791 & 0.664 \\
1 & 0 & 0.1 & 0.73 & -1.04771 & -1.049 & -0.224411 & -0.225 & 0.110292 & 0.117 \\
1 & 0.5 & 0.1 & 0.73 & -1.04771 & -1.049 & -0.224411 & -0.225 & 0.820805 & 0.825 \\
1 & 1 & 0.1 & 0.73 & -1.04771 & -1.049 & -0.224411 & -0.225 & 1.53188 & 1.533 \\
1 & 0.5 & 0.01 & 0.73 & -1.00398 & -1.005 & -0.230155 & -0.231 & 1.12575 & 1.125 \\
1 & 0.5 & 0.1 & 0.73 & -1.04771 & -1.049 & -0.224411 & -0.225 & 0.820805 & 0.825 \\
1 & 0.5 & 0.5 & 0.73 & -1.22325 & -1.234 & -0.204004 & -0.205 & 0.513629 & 0.514
\end{tabular}

Table 2: Variation of $f^{\prime \prime}, g^{\prime \prime}, \theta^{\prime}$ at the Plate Surface with F, Gr, M and Pr Parameters

\begin{tabular}{llllllllll}
\hline$F$ & $G r$ & $M$ & Pr & $\begin{array}{l}\text { Ahmed } \\
\mathrm{Y}[15] \\
g^{\prime \prime}(0)\end{array}$ & $\begin{array}{l}g^{\prime \prime}(0) \\
\text { Presen } \\
\mathrm{t}\end{array}$ & $\begin{array}{l}\text { Ahmed } \\
\mathrm{Y}[15]\end{array}$ & $\begin{array}{l}\theta^{\prime}(0) \text { Prese } \\
\theta^{\prime}(0)\end{array}$ & $\begin{array}{l}\text { Ahmed } \\
\mathrm{Y}[15]\end{array}$ & $\begin{array}{l}f^{\prime \prime}(0) \\
\text { Present } \\
f^{\prime \prime}(0)\end{array}$ \\
\hline 1 & 0.5 & 0.1 & 0.73 & -1.04771 & -1.049 & -0.224411 & -0.225 & 0.820805 & 0.825 \\
1 & 0.5 & 0.1 & 2 & -1.04771 & -1.048 & -0.480357 & -0.480 & 0.523724 & 0.531 \\
1 & 0.5 & 0.1 & 5 & -1.04771 & -1.048 & -0.882528 & -0.882 & 0.36651 & 0.374
\end{tabular}




\section{CONCLUSION}

In the present work we have studied the effect of porous media and radiation on MHD free convection flow near isothermal stretching sheet in the presence of a magnetic field. The effect of magnetic field reduces the temperature profiles and velocity profiles. It is observed that the effect of increasing porosity effect is leads to decreasing in the velocity profiles and increasing in temperature profiles. The velocity and temperature profiles decrease with the increasing radiation parameter $\mathrm{F}$. The velocity profiles increases with increase the parameter of relative difference between the temperature of the sheet, and the temperature far away from the sheet $r$.

\section{REFERENCES}

[1] Elbashbeshy,E.M.A.,Heat transfer over a stretching surface with variable surface heat

flux, J.Phys.D: Appl.Phys, 1998, Vol, 31,pp, 1951-1954.

[2] Gupta P.S. and Gupta, A.S.,Heat and mass transfer on a stretching sheet with suction

or blowing, Canadian

J.Chem.Engineering, 1977, Vol, 55,pp, 744746.

[3] Sakiadis, B.C, Boundary layer behaviour on continues solid surface: I. The boundary

layer equations for two - dimensional and axisymetric flow, 1961, AIChE J. Vol,7,

pp,26- 28.

[4] Takhar, H.S, Gorla, R.S.R and. Soundalgekar V.M, Radiation effects on MHD free convection flow of a gas past a semiinfinite vertical plate, International Journal of

Numerical Methods Heat Fluid Flow, 1966, Vol,6, no.2, pp, 77-83.
[5] Vajravelu,Kand.Hadyinicolaou, $A$

Convective heat transfer in a $n$ electrically

conducting fluid at a stretching surface

with uniform free stream

Internat.J.Engng.Science, 1997, Vol, 35 no. 12-13, pp, 1237-1244.

[6] Cortell, R.Effects of viscous dissipation and radiation on thermal boundary layer over

a nonlinearly stretching sheet, Phys. Lett.A 2008. Vol, 372, pp. 631-636.

[7] Cramer, K.R. and Pai, S. - I Magneto fluid dynamics for engineers and applied physics, Mc Graw hill books co., New York1973. pp. 204-237.

[8] Hayat, T., Z. Abbas, M.Sajid and S.Asghar, The influence of thermal radiation on

MHD flow of a second grade fluid, Int. J. Heat Mass Transfer, 2007b, Vol, 50, pp, 931-941,.

[9] Israel-cookey, C.,A. Ogulu and V.B. Omubo-Pepple, Influence of viscous dissipation and radiation on unsteady MHD free-convection flow past an Infinite heated vertical plate in a porous medium with time-dependent suction, Int.J. Heat 2003.

MassTransfer Vol, 642,pp, 305-2311,

[10] Mahmoud, M.A.A., Thermal radiation effects on MHD flow of a micropolar fluid

over a stretching surface with variable thermal conductivity, Physica A, 2007d.Vol, 375, pp, 401-410.

[11] Sajid, M. and T.Hayat, Influence of thermal radiation on the boundary layer flow

due to exponentially stretching sheet, Int.Comm. Heat Mass transfer, 2008, Vol, 35 ,

pp, 347-356, 
[12] Singh, A.K., K.S. Ajay and N.P.Singh, Heat and Mass transfer in MHD flow of a viscous fluid past a vertical plate under oscillatory suction velocity, Indian J.Pure Appl.Math. 2003, Vol, 34, pp, 429-442.

[13] Sondalgekar, V.M., Unsteady MHD free convection flow past an infinite vertical flat

plate with variable suction, Indian J.Pure Appl. Math. 1972, Vol, 3, pp, 426436,

[14] Ahmed Y. Ghaly and Elsayed M.E.Elbarbary, Radiation effect on MHD free Convection flow of a gas at a stretching surface with a uniform free stream, Journal of applied mathematics. 2002, Vol, 2.2, pp, 93-103,

[15] Rohsenow, W.M, .Hartnett, J.P and Y.I.Cho (eds), Hand book of heat transfer, $3^{\text {rd }}$

ed, McGraw-Hill, Newyork, 1998.

[16] Abo-Eldahab, E.M. and.Ghonaim, A.F, Radiationeffect on heat transfer of micropolar fluid through a porousmedium, Applied Mathematicsand Computation, Vol.169, no.1, pp, 500-510, 2005.

[17] Abo-Eldahab, E.M, Radiation effect on heat transfer in an electrically conducting fluid at a stretching surface with a uniform free stream, J.Phys.D:Appl.Phys. 2000. Vol. 33, pp, 3180-3185,

[18] Md.Abdus Samad and Mohd Mansur Rahman, Thermal radiation interaction with unsteady MHD flow past a vertical porous plate immersed in porous media,Journal of Naval Architecture and Marine
Engineering,June,2006Vol.3,pp, $\quad 7-$ 14.

[19] E.M.A Elbashbeshy, D.A.Aldawody, Effects of thermal radiation and magnetic field on unsteady mixed convection flow and heat transfer over a porous stretching surface, International Journal of Non-Linear Science, 2010, Vol 9No.4, pp 448454. 